\title{
Heterogeneous wireless sensor network routing protocol for an adaptive gray wolf optimizer
}

\author{
$1^{\text {st }}$ Chao Cheng ${ }^{12} ; 2^{\text {nd }}$ QingShan Han ${ }^{2} ; 3^{\text {rd }}$ GuoLi Cheng ${ }^{2} ; 4^{\text {th }}$ Shuang Zhai ${ }^{1}$ \\ ${ }^{1}$ School of Computer Science and Engineering, Changchun University of Technology, Changchun, China; \\ ${ }^{2}$ National Engineering Laboratory, CRRC Changchun Railway Vehicles Co., Ltd., Changchun, China; \\ $1^{\text {st }}$ ) chengchao@ @cut.edu.cn; $2^{\text {nd }}$ ) hany0301@163.com; $3^{\text {rd }}$ ) chengguoli@ cccar.com.cn; \\ $\left.4^{\text {th }}\right)$ zhaishuang @ ccut.edu.cn
}

\begin{abstract}
Wireless sensor network (WSN), plays an increasingly important role in information collection. In this paper, firstly, in order to adapt to the actual conditions, the communication process of the nodes energy is limited, and a three-stage energy heterogeneous network model is designed. Secondly, for the convergence node frequent task forwarding and complex cluster first-round energy consumption, by combining the optimal number of cluster heads with the gray wolf optimization algorithm, a new fitness function is designed that integrates the remaining energy of the nodes and the distance from the nodes to the base station. In addition, an improved iterative factor is introduced to enhance the ability of local search in cluster head selection, so as to improve the accuracy of cluster head search. Finally, the simulation results show that the proposed method extends the lifetime of the network 50\%, reduces the process of energy consumption, and improves the throughput of network data $30 \%$.
\end{abstract}

Keywords: Energy efficient; gray wolf optimizer (GWO); balanced cluster structure; wireless sensor network

\section{Introduction}

With the development of wireless communication technology and sensor technology, the self-organizing wireless sensor network with micro and low-power nodes plays an increasingly important role in information acquisition under the special circumstances [1].The WSN nodes, which is randomly monitored around the clock, is widely used in the fields of environmental monitoring, military reconnaissance and medical data collection [2-4]. Because the nodes in WSN are powered by a limited capacity micro-battery, it is a hot issue to find a WSN routing protocol with high energy utilization and strong fault tolerance to reduce the energy loss of the node, thus prolonging the network life cycle [5-7].

The WSN routing model can be divided into isomorphic networks and heterogeneous networks according to the consistency of sensor type and communication radius distance [8,9]. One of the most representative routing protocol is the Low Energy Adaptive Clustering (Leach) [10] proposed by Heinzelman in 2000. The algorithm takes the dynamic "wheel" as the working period, and randomly selects the cluster heads $(\mathrm{CHs})$ by setting the threshold and the probability combination, but the probability of the lower energy node being selected as the $\mathrm{CHs}$ is the same as the probability of the normal node. In order to improve the simple probability to determine the energy consumption, many scholars have designed quite a few 
new protocols to improve the performance of LEACH [11-12] by reducing the energy consumption of the CHs. In [13], the LEACH-EA algorithm, which uses enhanced network communication to reduce energy consumption, improves the survivability of the first node in the literature. However, the way in which cluster heads are selected based on probability does not change. In [14], an energy balance hierarchical routing algorithm (EBHRA) is proposed, which uses the residual energy of the node as the CHs election weight.However, the method of considering only the remaining energy of the node is too single. The possibility of CHs increases the energy loss of long-distance communication networks. A multi-factor cluster head selection strategy for residual energy and node density decision factors is proposed in the literature [15], which improves the decision factor of cluster head, but improves the network survival cycle by about $10 \%$ to $60 \%$. In order to improve the traditional node clustering method, Tapswini Samant et al. proposed to reduce the number of data transmissions and reduce the network energy consumption low energy threshold sensitivity protocol (TEEN) by setting the node sensing threshold [16]. Since cooperative communication requires multiple nodes, the TEEN protocol is mainly used for cluster formation. On this basis, the TEENvector quantization (TEEN-V) protocol proposed by D.E. Boubiche et al., which is used for cluster communication in cooperative networks to improve the survival period of the network as much as possible [17]. However, since the ideal homogeneous network structure does not exist under realistic conditions, the homogeneous network model has great limitations in the process of production practice.

Therefore, it is important to design a heterogeneous WSN network with high reliability, integrity of data transmission, and enhanced heterogeneity of nodes $[18,19]$. Smaragdakis et al. proposed the earliest heterogeneous stable election protocol (SEP) to extend the death period of the first node in the network [20], but did not consider the residual energy and node location information of the node. A multi-hop routing communication protocol (MCR) is proposed in the literature [21], which changes the election mode of $\mathrm{CHs}$ by reducing network stability, but the energy level is too simple to set the level to achieve the complexity of calculating heterogeneous. Young et al. proposed a distributed energy efficient clustering (DEEC) network heterogeneous model, which selects the $\mathrm{CHs}$ by the ratio of the residual energy of the surviving node in the network to the average energy of the entire node [22]. If only from the perspective of energy consumption, when the $\mathrm{CHs}$ are far away from the base station BS node, there is still a chance to be elected cluster heads to undoubtedly increase the network energy burden; In [23-24], an enhanced DEEC algorithm (E-DEEC) for enhanced heterogeneous LEACH (EHE-LEACH) and three nodes was proposed.Therefore, the importance of routing node density and super node to enhance the stability and heterogeneity of the network life cycle is emphasized. In [25], Ya liang et al. combined heterogeneous data with tensor multi-clustering method (TMC) to provide a future research value for evaluating the cluster performance of data. The main contributions of this paper are as follows:

- According to the energy model in the wireless sensor network, the optimal number of cluster heads is selected. Combined with the K-MEANS algorithm to complete the process of starting clustering, reduce the energy loss in the process of cluster establishment.

- Adopting the Gray-wolf Algorithm decision-making model of sub-region, combining the energy heterogeneity and computing heterogeneous characteristics of heterogeneous wireless sensor networks to select the $\mathrm{CHs}$ candidate nodes that are most suitable for the network.

- Construct a information evaluation model that combines the distance from node to the base station and the node's own energy, introduces the adaptive factor of local 
optimization of weight renewal, enhances the local optimization mechanism of the algorithm, and finds the most suitable cluster head.

The rest of the paper is orgainzed as follows. In Section II mainly introduces the energy model in the wireless sensor network, which is the basis of the design of this paper. In Section III proposes an adaptive gray wolf algorithm routing decision model, and the Section IV is to verify the simulation results. Finally, the conclusion are drawn in Section V .

\section{Network and energy model}

According to the heterogeneous characteristics of sensor nodes, heterogeneous WSNs can be classified three types: energy heterogeneity, heterogeneous communication capability and heterogeneous computing power. Energy heterogeneous means that network node configurations have different initial energy; Heterogeneous communication capability is the difference between node transmission rate, communication link and communication protocol. the computing capability is heterogeneous in the node processing processing capability, sensing capability and storage space difference. In heterogeneous WSNs, whether the communication capability is heterogeneous or the computing power is heterogeneous, as the network operation will lead to node energy heterogeneity, energy isomerism is the basis for studying heterogeneous WSN.

The energy consumption model of the first-order wireless communication mode used in this paper is shown in Figure 1. The energy of the nodes in the model is limited, and the radio signals consume the same initial energy in all directions [26].
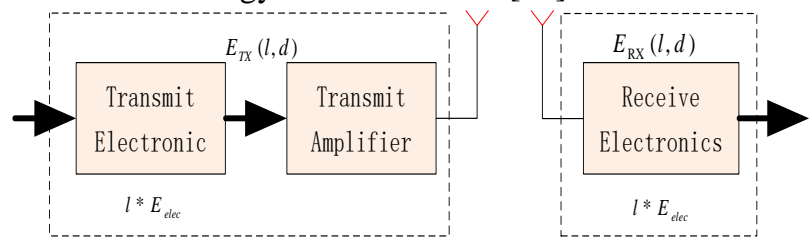

Fig. 1.Energy dissipation model

According to the energy loss model, the energy consumed when transmitting 1-bit information is shown as [27]:

$$
E_{T X}(l, d)=E_{T X-\mathrm{elec}}(l)+E_{T X-m p}(l, d)= \begin{cases}l * E_{\mathrm{elec}}+l^{*} \varepsilon_{m p} * d^{4} & \left(d \geq d_{0}\right) \\ l * E_{\mathrm{elec}}+l^{*} \varepsilon_{f s} * d^{2} & \left(d<d_{0}\right)\end{cases}
$$

where $E_{T X-\text { elec }}, E_{T X-\mathrm{mp}}$ is the energy consumed by the transmitter when transmitting or receiving 1 bit of data, $\varepsilon_{\mathrm{mp}}$ is the power amplification factor of the multipath attenuation model, and $\varepsilon_{\mathrm{fs}}$ is the power amplification factor of the free space model. When the transmission distance $d \geq d_{0}$.

$$
d_{0}=\frac{\sqrt{\varepsilon_{f s}}}{\sqrt{\varepsilon_{m p}}}
$$




\section{Routing decision based on adaptive grey wolf algorithm (AD-GWO)}

The three most suitable sensor nodes are selected by designing a combination of node residual energy and node-to-base station distance characteristics. Give the three nodes the strongest guiding effect, guiding the evolution direction of the whole population, thus approaching the best cluster head position. In order to reduce the time and space loss of the iterative process, an adaptive iterative factor selection method is proposed. In order to reduce the energy loss of nodes joining the cluster network in the random clustering process, selecting the appropriate $\mathrm{CHs}$ is an indispensable process for establishing a heterogeneous network. The specific analysis is as follows:

\subsection{Energy-based optimal cluster head number selection decision}

According to the physical protocol and the running process, the main energy consumed by the $\mathrm{CHs}$ is mainly divided into the energy consumed in the data acquisition phase, the energy in the data capacity and the processing phase. the free decay model is mainly used in this paper. Assuming that $\mathrm{N}$ random nodes are randomly deployed in an $\mathrm{M} * \mathrm{M}$ region, the average number of non-cluster head members in each cluster is $\mathrm{N} / \mathrm{K}-1$ [28], and the energy consumption of the $\mathrm{CHs}$ is as shown:

$$
E_{C H}=l * E_{\text {elec }} *\left(\frac{N}{K}-1\right)+l * E_{D A} * \frac{N}{K}+\left(l * E_{\text {elec }}+l * \varepsilon_{f s} * d_{\text {toBS }}^{2}\right)
$$

The non-CHs consumes energy as shown in equation (4)(5):

$$
\begin{gathered}
E_{N C H}=l^{*} E_{\text {elec }}+l^{*} \varepsilon_{f s} * d_{\text {toch }}^{2} \\
E\left[d_{\text {toch }}^{2}\right]=\iint\left(x^{2}+y^{2}\right) \rho(x, y) d x d y=\frac{M^{2}}{2 \pi K}
\end{gathered}
$$

Then the total energy consumed in a cluster.

$$
E_{c l u}=E_{C H}+\sum E_{N C H}=E_{C H}+\left(\frac{N}{K}-1\right) * E_{N C H}
$$

Then the total energy consumed in the K clusters is:

$$
E_{r}=K * E_{c l u}=l *\left[2 E_{\text {elec }} * N+N * E D A+k * \varepsilon_{m p} * d_{t o B S}^{4}+N * \frac{\varepsilon_{f s} * M^{2}}{2 \pi K}\right]
$$

If the energy consumption in each of the above equations is regarded as a function of $\mathrm{K}$ [29], then:

$$
K=\sqrt{\frac{\varepsilon_{f s} * N * M^{2}}{2 \pi * \varepsilon_{m p}}} * \frac{1}{d_{t o B S}}
$$

In the above formula, the simulation results in Chapter Section IV show that when the base station is located at $(50,50), d_{t o B S}$ is about $141 \mathrm{~m}$, and according to the minimum of $50 \mathrm{~m}$ in the literature, the value of $\mathrm{K}$ in this paper is in [4,7].According to the simulation results, the following table can be obtained:

The $(\mathrm{CHs})$ receives information by all $\mathrm{CNs}$ members in the cluster, and transmits collects information to the base station(BS) through data fusion. However, the distance $d_{c b}$ (CHs to the BS) is not same, and the process of energy consumption of the $\mathrm{CHs}$ determines the robustness of entire network. If $N_{u m b e r}{ }_{c h s}<K(\mathrm{~K}$ is the number of $\mathrm{CHs}$ actually needed), the 
CHs cannot cover all the monitoring areas; and the energy loss during data transmission will increase accordingly. Therefore, it is especially important to choose the appropriate CHs.

Table 1. The survival time corresponding to different $\mathrm{K}$ values

\begin{tabular}{cccc}
\hline K value & Number & Initial node death & All nodes die \\
\hline \multirow{3}{*}{$\mathrm{K}=5$} & 1 & 1261 & 2033 \\
& 2 & 1286 & 2053 \\
& 3 & 1047 & 2059 \\
\hline \multirow{3}{*}{$\mathrm{K}=6$} & 1 & 1060 & 2030 \\
& 2 & 968 & 1998 \\
& 3 & 928 & 2013 \\
\hline \multirow{2}{*}{$\mathrm{K}=7$} & 1 & 998 & 2018 \\
& 2 & 993 & 2016 \\
\hline
\end{tabular}

Different from the traditional WSN network establishment process, this paper first compares the distance between each node and BS. If the basic communication requirements are met, direct communication is performed. Otherwise, the distance from each node to the $\mathrm{CHs}$ will enter the cluster establishment phase. This approach reduces the energy consumption during the setup phase of the clusters complex clusters. The detailed pseudo code is shown in Algorithm 1:

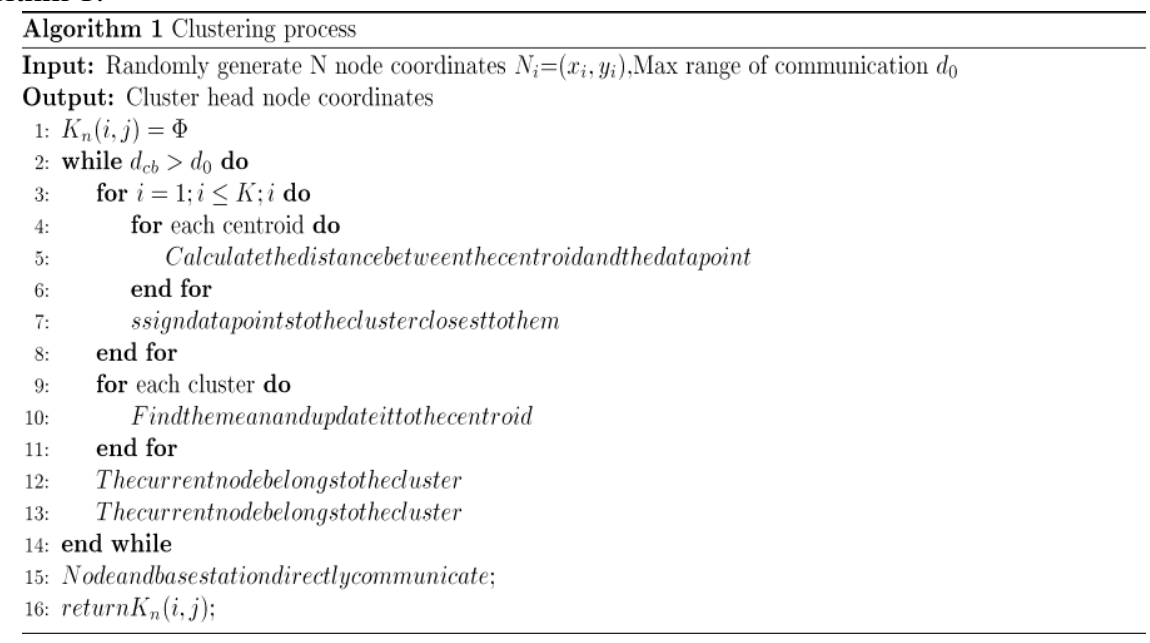

\subsection{Adaptive Grey Wolf Optimization Algorithm (Ad-GWO) Model}

As a typical cluster intelligent optimization algorithm, GWO [30] simulates the predation behavior of the grey wolf population, and compares the wolf group tracking process with the prey as the optimization process, so as to achieve the optimal solution. Applying GWO to the cluster head optimization problem of WSNs, the position of the wolf group represents the position of the sensor. Compared with the position where the prey is hunt, the position of the $\mathrm{CHs}$ is also the position of the optimal solution expressed in this paper. In the gray wolf algorithm, the $\alpha$ wolf, the $\beta$ wolf, and the $\varepsilon$ wolf represent the first dominant node the second dominant node, and the third dominant node in the wireless sensor network. They are at the 
top of the population, and have the best decision-making ability compared to the common wolves. Other wolves must obey the instructions of the highest priority first dominant node compared to the three highest priority sensor nodes. According to the existing algorithm, the GWO algorithm is mainly divided into two main processes of prey and hunting [31-33]. The distance between the prey and the wolves needs to be ascertained in the enveloping process as follows:

$$
\vec{D}=\left|C \overrightarrow{X_{p}^{t}}-\overrightarrow{X^{t}}\right|
$$

where $\vec{X}_{p}^{t}$ is the position of the prey when iterating to $\mathrm{t}^{t h}, \vec{X}^{t}$ is the position of the gray wolf at this moment, $\mathrm{C}$ is a constant factor, so the following formula.

$$
C=2 * r_{1}, r_{1} \in[0,2]
$$

Then according to the surrounding prey information, we can calculate the specific direction of the wolves in the next step, then the next round of wolves is:

$$
\overrightarrow{X^{t+1}}=\left|\overrightarrow{X^{t}}-S \vec{D}\right|
$$

in which $\mathrm{S}$ is the convergence factor expressed by the following formula:

$$
S=2 * s * r_{2}-s, r_{2} \in[0,2]
$$

The hunting process in the traditional algorithm will directly determine the final position of the three wolves with the highest priority, and the expression will be brought into the expression of Eq. 11 as:

$$
\begin{aligned}
& \overrightarrow{X_{\alpha+1}^{t+1}}=\overrightarrow{X_{\alpha}^{t}}-\vec{S}_{1} * D_{\alpha} \\
& \vec{X}_{\beta+1}^{t+1}=\overrightarrow{X_{\beta}^{t}}-\vec{S}_{1} * D_{\beta} \\
& \overrightarrow{X_{\varepsilon+1}^{t+1}}=\overrightarrow{X_{\varepsilon}^{t}}-\vec{S}_{1} * D_{\varepsilon}
\end{aligned}
$$

Then the best cluster head position is available:

$$
X_{p}{ }^{t+1}=\frac{\overrightarrow{X_{\alpha}^{t+1}}+\overrightarrow{X_{\beta}^{t+1}}+\overrightarrow{X_{\varepsilon}^{t+1}}}{3}
$$

\subsubsection{Ray wolf fitness function model based on energy and distance}

According to the energy consumption model established in Section 2, When the distance between the node and the base station is greater, the energy consumed by the node will also be greater, and whether the current node has the opportunity to become a cluster head is based on the residual energy of the current node. Selecting nodes with high residual energy helps to improve the survival time of the network and prevent the occurrence of energy holes. Therefore, this paper designs an adaptive value function based on the residual energy of the node and the communication distance between the node and the BS as follows:

$$
F=\left\{\begin{array}{cc}
\gamma * \frac{E_{i}}{E_{r}}+\lambda * \frac{D_{i}}{D_{\text {Avg }}} & E_{i} \neq 0 \\
0 & E_{i} \leq 0
\end{array}\right.
$$


In the above formula, both $\gamma$ and $\lambda$ are influence factors, ignoring other losses in the transmission process. It is assumed that the cluster head is only affected by both distance and energy, and the sum of the influence factors is $1 . E i$ is the current node energy, $E r$ is the total energy in the current cluster, $D i$ is the distance from the current node to the base station, and $D_{\text {Avg }}$ is the average distance from the node in the current cluster to the base station. At the same time, in order to emphasize the position of the first dominant node, the second dominant node and the third dominant node of the wireless sensor network, the position of the cluster head node in the whole heterogeneous network is determined, and the optimal weighting factor is re-set, and the improved most The location of the cluster head is as follows:

$$
\begin{aligned}
& E_{\mathrm{r}}=\sum_{i=1}^{n} E_{i} \quad(E i \geq 0) \\
& \left\{\begin{array}{l}
F_{\alpha}=\frac{F_{\alpha}}{F_{\alpha}+F_{\beta}+F_{\delta}} \\
F_{\beta}=\frac{F_{\beta}}{F_{\alpha}+F_{\beta}+F_{\delta}} \\
F_{\delta}=\frac{F_{\delta}}{F_{\alpha}+F_{\beta}+F_{\delta}}
\end{array}\right.
\end{aligned}
$$

\subsubsection{An improved convergence factor adaptive adjustment strategy}

According to the design principle of the algorithm, in the early iteration, the convergence speed of the function is faster. As the number of iterations increases, the iteration speed of the algorithm gradually decreases. It can be seen from the above evidence that the iteration factor $S$ plays a key role in the degree of convergence of the algorithm. In this paper, the nonlinear adjustment strategy of a cosine function is used to extend the original $s \in[0,2]$ interval to the tradition GWO algorithm based on the nonlinear reduction of the cosine function on $[0, \pi / 2]$ :

$$
s=2 * \cos \left(\frac{t}{t_{\max }} * \frac{\pi}{2}\right)
$$

Where $t_{\max }$ is the maximum number of iterations and $\mathrm{t}$ is the current number of iterations. The fitness value of each wireless sensor network node in each cluster can be found from the adaptation value in Eq.15. Comparing each adaptation value $F i$ with the average fitness value, If the self-adaptation value is higher than the average fitness value, then the individual should be adjusted to the direction of the prey in the hunt. Otherwise, the influence range of the control parameters should be expanded to enhance the global search interval as shown below.

$$
s=\left\{\begin{aligned}
2 * \cos \left(\frac{t}{t_{\max }} * \frac{\pi}{2}\right) & F_{i} \geq F_{A v g} \\
-2 t+2 & F_{i}<F_{A v g}
\end{aligned}\right.
$$

The detailed pseudo code for Ad-GWO is as follows: 


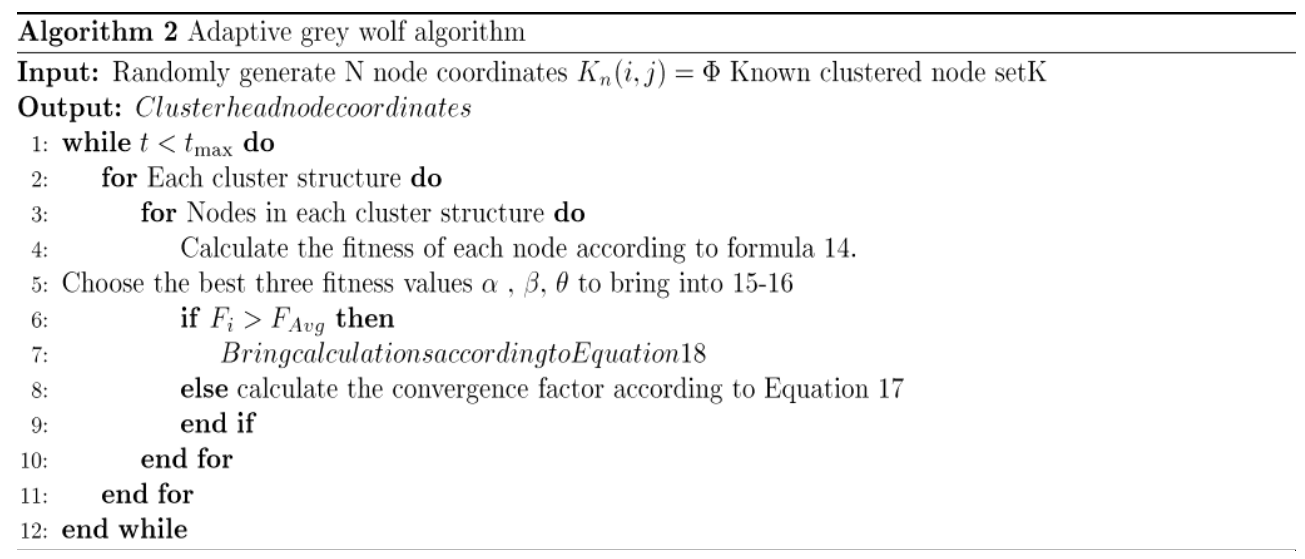

\section{Simulation results}

In order to evaluate the algorithm of this paper and the performance indicators of existing algorithms, this paper proposes a variety of simulation evaluation methods. In the simulation area of $100 \mathrm{~m}^{*} 100 \mathrm{~m}, 100$ nodes are randomly thrown around the BS node located in the central area, and a three-level energy heterogeneous network is constructed as shown in Figure. 2;Among them, $40 \%$ of the energy is deployed as the ordinary node, and the E0 level node is represented by 'o'; A three-level energy node with $20 \%$ energy of $1 / 2 E_{0}$ is deployed, denoted by ' + ', the remaining nodes are normal secondary nodes, denoted by '*', and the BS node is denoted by ' $\mathrm{X}$ '. In the formulas 1 to 8 , the optimal $\mathrm{K}$ value is derived. In this paper, the $\mathrm{K}$ means algorithm is used to cluster the unordered network nodes, and the clustering strategy is adopted to help determine the cluster head node and the base station. Minimum distance between, reduce network energy consumption, and the final clustering result is represented by Tyson polygon as shown in Figure 3.

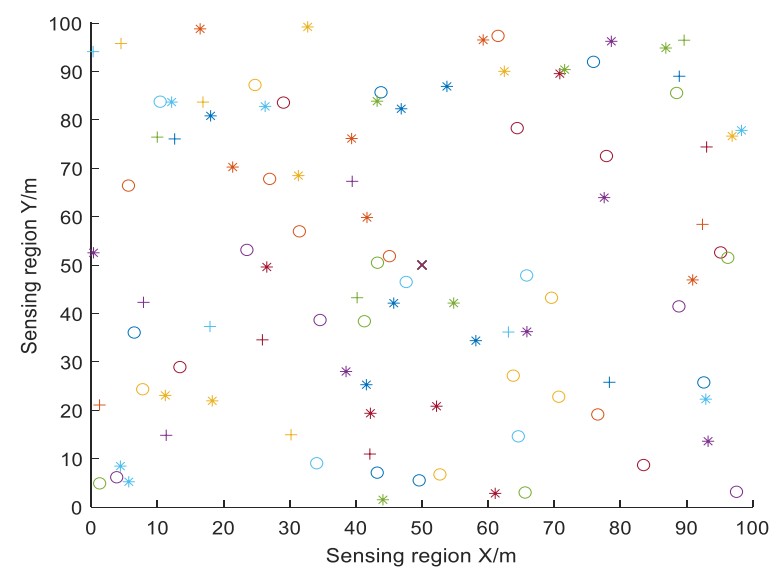

Fig. 2. Energy dissipation model. 


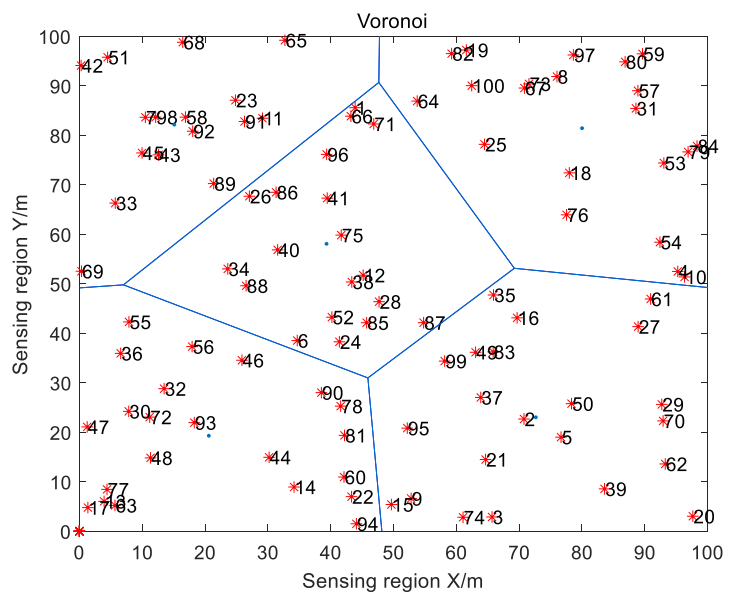

Fig. 3. K-means clustering algorithm results

In the test to simulate the effect of the AD-GWO algorithm, the initial experimental parameters determine the performance of the entire experimental results. The specific parameters are shown in the Table 2 below. Since the unreasonable energy allocation and consumption protocol accelerates the process of node death, the more energy the node stores, it means that the benefits of maximizing network survival directly reflect the survival value of the agreement.

Table 2. Key parameters.

\begin{tabular}{cc}
\hline Parameter & Font size and style \\
\hline Network Size & $\left\{100^{2}\right\}$ \\
Number of Sencer nodes & 100 \\
Protion of CHs & Table 1 \\
Data Aggregation Energy Cost & $\mathrm{E}_{\mathrm{DA}}=50 \mathrm{~nJ} / \mathrm{bit}$ \\
Transmitter/Receive & $\mathrm{E}_{\mathrm{elec}}=50 \mathrm{~nJ} / \mathrm{bit}$ \\
Packet Size & $4000 \mathrm{bits}$ \\
Transmitter Amplifie & $0.0013 \mathrm{pJ} /\left(\mathrm{bit} \cdot \mathrm{m}^{4}\right.$ \\
Transmitter Amplifier & $0.0013 \mathrm{pJ} /\left(\mathrm{bit} \cdot \mathrm{m}^{4}\right)$ \\
$\lambda$ & 0.7 \\
\hline
\end{tabular}

\subsection{Residual energy balance ratio(REB)}

The remaining energy balance ratio(REB) is expressed as the ratio of the remaining energy of the current node to the total energy of the node, since the initial energy priorities are different, the energy loss values of the various nodes are different at different locations. Figure 4 shows that the AD-GWO algorithm proposed in this paper has four dead nodes in 1000 iterations, and the REB ratio of most nodes exceeds $50 \% \mathrm{E}_{0}$; Figure 5 is the energy balance ratio of the improved heterogeneous LEACH (HLEACH-e) algorithm under the same number of iterations. In this mode, there are 59 dead nodes, and most of the REB ratio are between $[40 \%, 50 \%] \mathrm{E}_{0}$; In Figure 6, the number of dead nodes based on the improved heterogeneous 
SPE algorithm is about 11 , and the REB ratio is between [20\%,60\%] $\mathrm{E}_{0}$; In Figure 7, the energy nodes of the heterogeneous Fuzzy C-means clustering DEEC(FCM-DEEC) die are about 11 , but most of the energy nodes of the remaining nodes are distributed between $[20 \%, 40 \%] \mathrm{E}_{0}$; And a considerable number of nodes are dying, so it can be clearly seen from the above data that the proposed adaptive gray wolf algorithm has significant effects on solving the energy balance problem in heterogeneous wireless sensor networks.

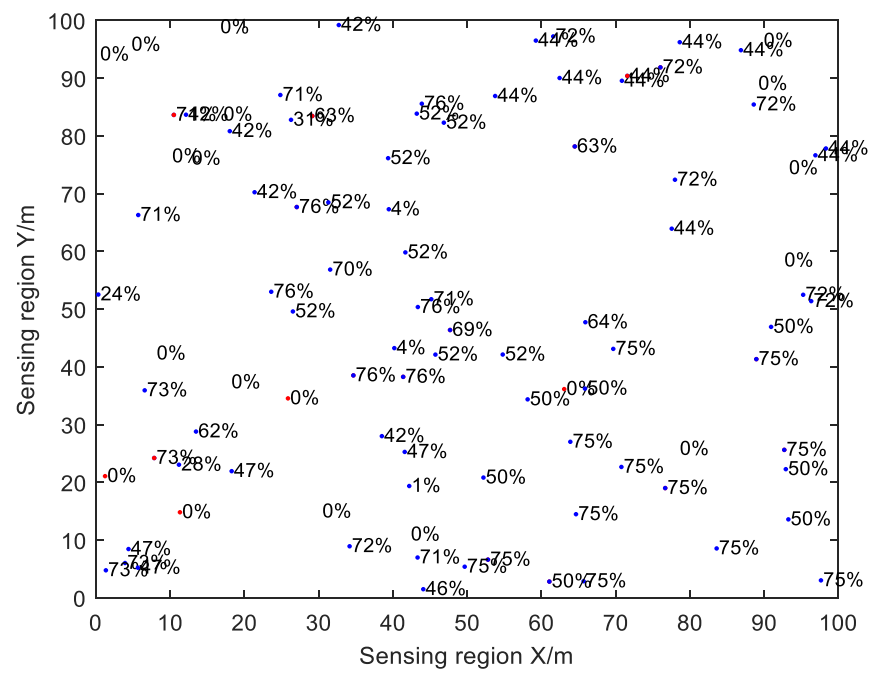

Fig.4. AD-HGWO 1000 generation energy balance ratio.

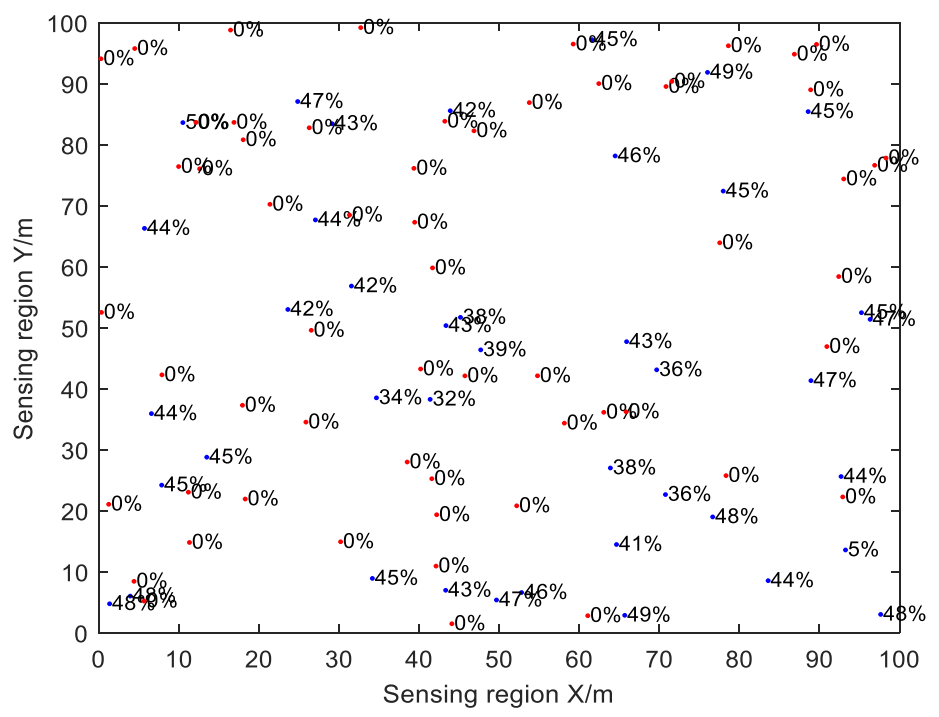

Fig.5. HLEACH-e 1000 generation energy balance ratio. 


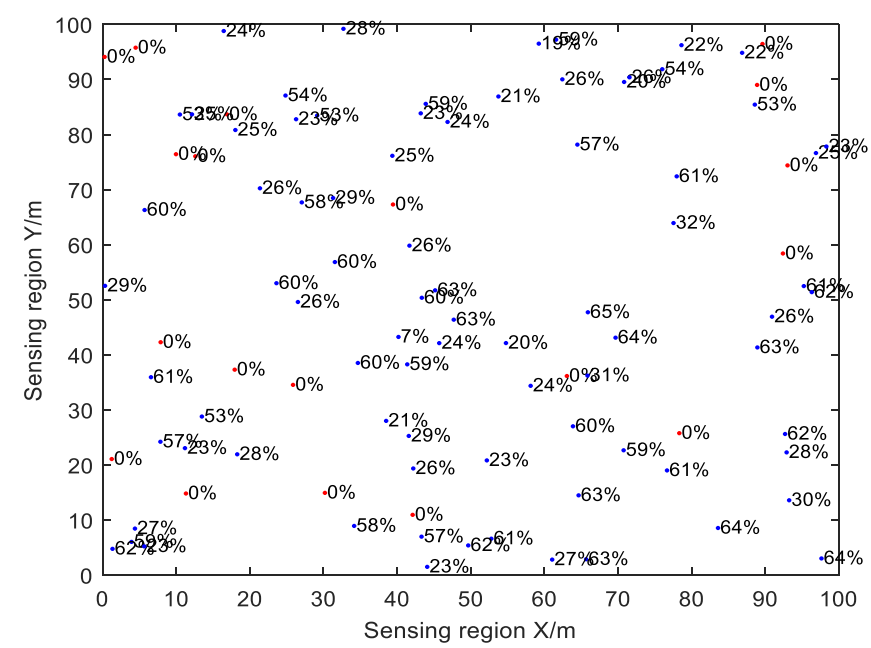

Fig.6. FCM-DEEC algorithm 1000 generation energy balance ratio

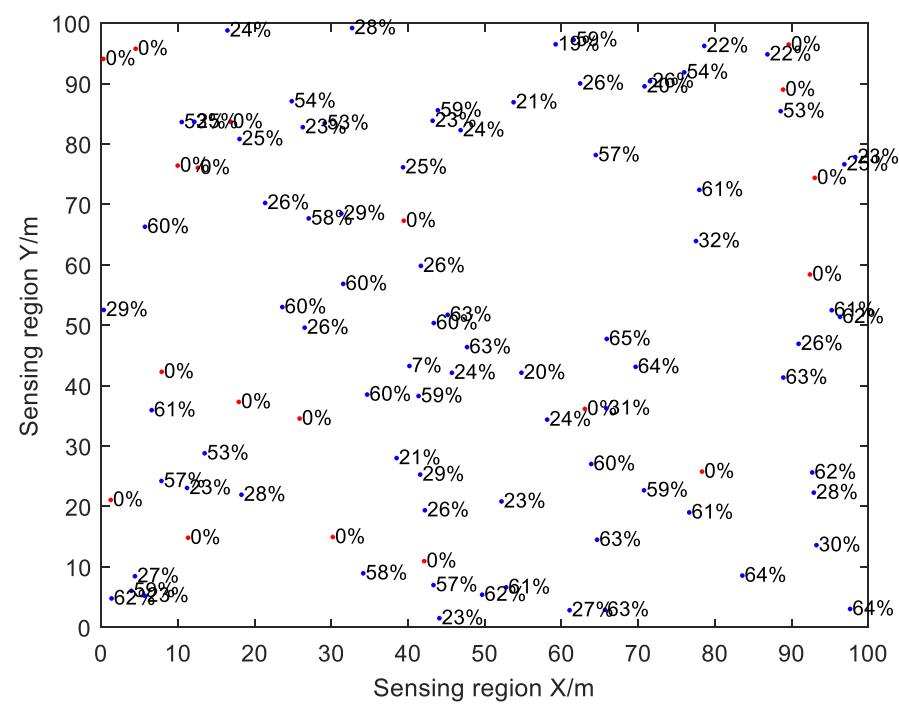

Fig.7. SEP algorithm 1000 generation energy balance ratio

\subsection{Node life cycle}

In the heterogeneous wireless sensor network, due to the large difference of the three levels of energy, the improved LEACH algorithm highlights the serious first node death based on the probability disadvantage; Although the improved FCM-HDEEC algorithm makes up for this deficiency, the problem of cluster head selection does not essentially solve the inherent defects in the algorithm process; The SPE algorithm itself adopts two 
nodes with different initial energy, and designs different cluster head election thresholds, which further increases the probability that the advanced node becomes the cluster head, and improves the death time of the first node; However, this approach does not take into account the negative impact of the cluster head node and base station distance. The probability of death of the node in a certain period of time is further increased, and the lifting effect is not obtained. The simulation results are shown in Figure 8:

Table 3. The survival time corresponding to different $\mathrm{K}$ values

\begin{tabular}{cccc}
\hline Algorithm & Initial Node Died & Half Node Died & All Node Died \\
HLEACH-e & 386 & 936 & 2131 \\
SEP & 813 & 1086 & 1352 \\
FCM-DEEC & 820 & 1515 & 2805 \\
AD-HGWO & 500 & 1995 & 3972 \\
\hline
\end{tabular}

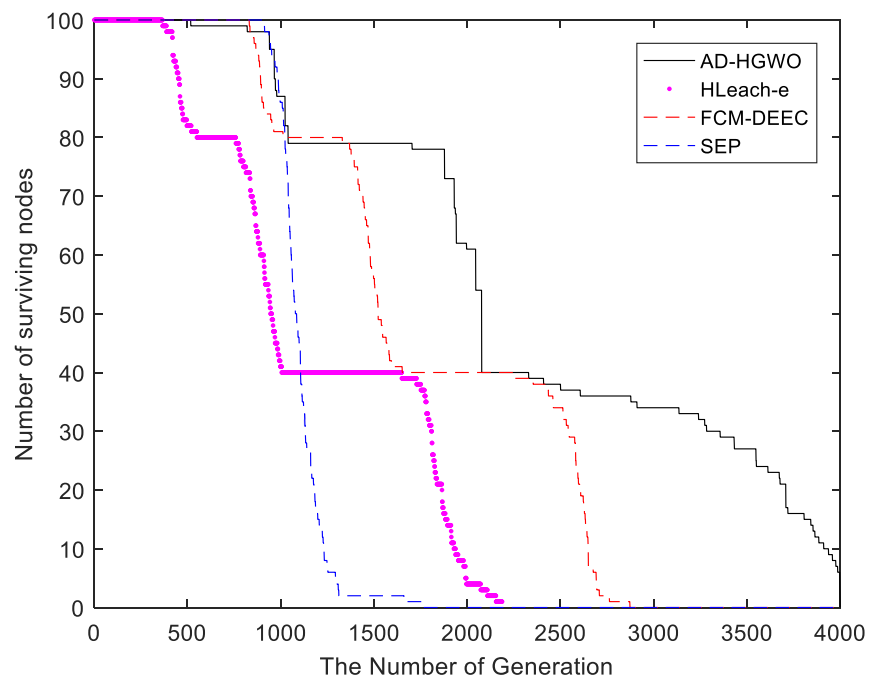

Fig.8. Stability period with respect to number of rounds

In Table 3, the initial node death algebra, $50 \% \mathrm{E}_{0}$ node death algebra, and all node death algebras of various algorithms are Therefore, this paper has profound significance and influence in improving the network life cycle.

\subsection{Number of data packets received by the base station}

The more data received by the base station, the longer the survival time of the network, which means that the amount of information monitored by the network will be larger in complex environments. This indicator directly reflects the effectiveness of the proposed algorithm. As shown in figure 9, the data in this paper is about 36679 , which is $65 \%, 49 \%$, and $50 \%$ improved by HLEACH-e algorithm, FCM-DEEC algorithm, and SPE algorithm respectively. 


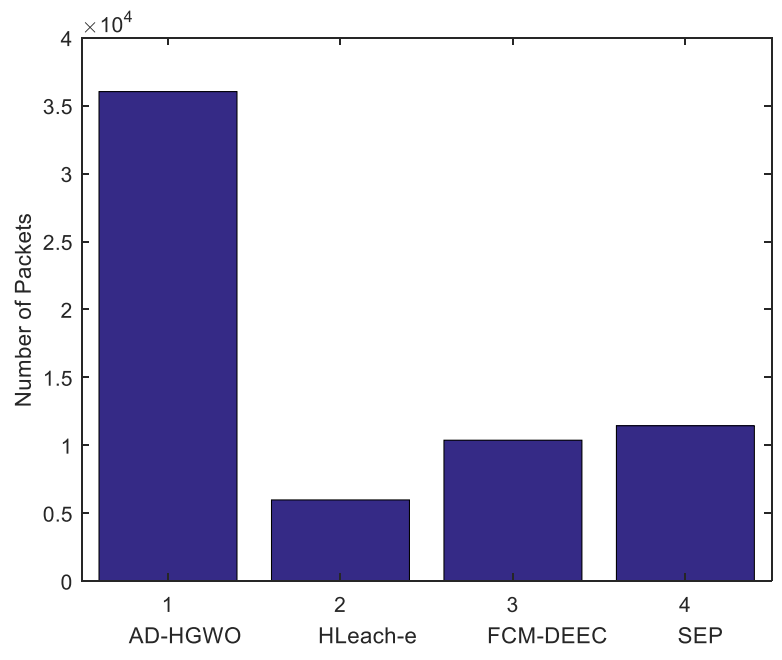

Fig.9. Base station receives data value

The average node residual energy is the ratio between the total energy of the current node and the number of current nodes. When the average node residual energy means the higher the survival value of the network, the greater the use value of the network. In the algorithm proposed in this paper, the slope is the smallest compared to the other three algorithms, which means that the energy loss in the same network living space is slower. It is about 33\%higher than the HLEACH-e algorithm and FCM-DEEC algorithm, and nearly50\% higher than the SPE algorithm. The method proposed in this paper saves the total energy consumption of the network and improves the energy utilization of a single node.

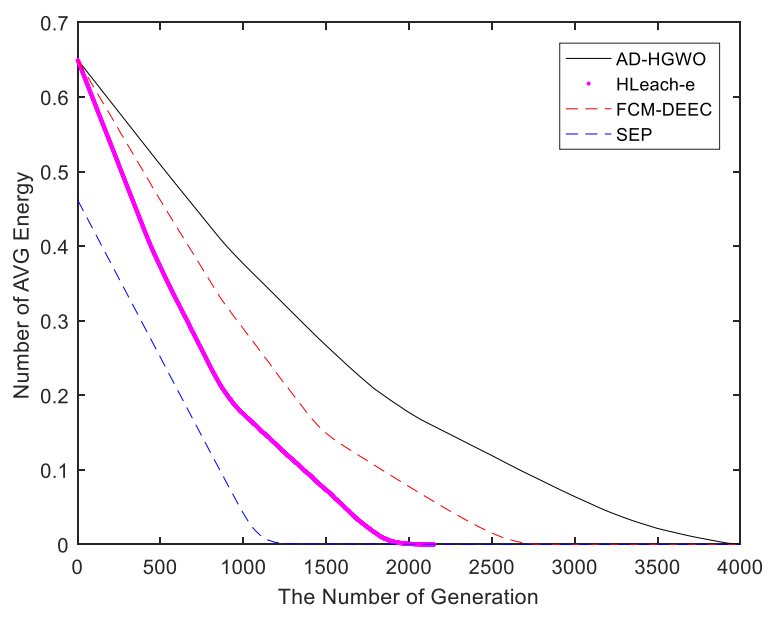

Fig.10. Average residual energy 


\section{Conclusion}

This paper proposes an adaptive gray wolf algorithm for the three-level energy heterogeneous wireless sensor network, which improves the network life cycle and improves the network residual energy usage rate. Firstly, a reasonable clustering structure is set by the energy consumption model of the wireless sensor network. The K-means algorithm is used to ensure that the nodes in each cluster are in a reasonable range, and the irrelevant energy loss of the cluster members in selecting the unreasonable cluster head is avoided. Secondly, the fitness function is established by combining the residual energy of the network node with the distance from the node to the base station. Make full use of the relationship between node energy and position in the information transfer process, combined with the logical composition of the gray wolf algorithm, select the three most adaptable nodes in each cluster, and iteratively select the cluster head nodes that best reflect the current cluster structure. Complete the information of the clustered transfer sensor; finally, simulate the real network environment by using various evaluation criteria. The experimental results show that the model has a good network life cycle and has better adaptability than the traditional homogeneous and heterogeneous network models. In addition, although the proposed algorithm improves the life cycle of heterogeneous WSN networks, it increases the computational complexity of the algorithm. The base station and the network node of the algorithm are not mobile, and the delay and jitter in the information transmission process are not considered. Therefore, future work can further consider the scope of adaptation of the algorithm.

\section{References}

[1] J. Zhu, C. Gong, S. Zhang, M. Zhao and W. Zhou: Foundation study on wireless big data: Concept. mining learning and practices.China Communications.pp. 1-15, (2018) .

[2] Y. Ahmadi, N. Neda and R. Ghazizadeh:Range Free Localization in Wireless Sensor Networks for Homogeneous and Non-Homogeneous Environment.IEEE Sensors Journal. pp. 8018-8026(2016) .

[3] M. Tokala and R. Nallamekala:Secured algorithm for routing the military field data using Dynamic Sink:WSN.2018 Second International Conference on Inventive Communication and Computational Tech nologies (ICICCT). pp. 471-476(2018) .

[4] D. Abbasinezhad-Mood and M. Nikooghadam:Efficient Design of a Novel ECC-Based Public Key S cheme for Medical Data Protection by Utilization of NanoPi Fire.IEEE Transactions on Reliability. pp. 1 328-1339(2018).

[5] O. J. Pandey and R. M. Hegde.Low-Latency and Energy-Balanced Data Transmission Over Cognitiv e Small World WSN.IEEE Transactions on Vehicular Technology.pp. 7719-7733(2018)

[6] Z. Zhou, C. Du, L. Shu, G. Hancke, J. Niu and H. Ning:An Energy-Balanced Heuristic for Mobile Si nk Scheduling in Hybrid WSNs.IEEE Transactions on Industrial Informatics. pp. 28-40, Feb( 2016).

[7] O.Ogundile, M. B. Balogun, O. E. Ijiga and E. O. Falayi.Energy-balanced and energy-efficient clust ering routing protocol for wireless sensor networks.IET Communications pp. 1449-1457(2019) .

[8] J. Zhang and R. Yan.Centralized Energy-Efficient Clustering Routing Protocol for Mobile Nodes in Wireless Sensor Networks.IEEE Communications Letters. pp. 1215-1218(2019) .

[9] J. Leu, T. Chiang, M. Yu and K. Su:Energy Efficient Clustering Scheme for Prolonging the Lifetime of Wireless Sensor Network With Isolated Nodes.IEEE Communications Letters. pp. 259-262(2015) .

[10]S. Gupta and N. Marriwala.Improved distance energy based LEACH protocol for cluster head electi on in wireless sensor networks.2017 4th International Conference on Signal Processing, Computing and Control (ISPCC). pp. 91-96(2017).

[11]R. Gantassi, A. Ben Yagouta and B. Ben Gouissem:Improvement of the LEACH protocol in load bal ancing and energy-association conservation.2017 International Conference on Internet of Things, Embed ded Systems and Communications (IINTEC). pp. 53-59(2017). 
[12]K. Qian:An Energy Balanced Wireless Sensor Network Hierarchical Routing Algorithm.2013 Intern ational Conference on Computer Sciences and Applications. pp. 154-157(2013).

[13]Q. Li, J. Sun and D. Lu:Adaptive Unequal Clustering Using an Improved LEACH Protocol with Ene rgy Balance.2018 IEEE 18th International Conference on Communication Technology (ICCT). pp. 528532(2018).

[14]T. M. Behera, U. C. Samal and S. K. Mohapatra:Energy-efficient modified LEACH protocol for IoT application.IET Wireless Sensor Systems.pp. 223-228(2018).

[15]S. K. Singh, P. Kumar and J. P. Singh:A Survey on Successors of LEACH Protocol.IEEE Access, p p. 4298-4328(2017).

[16]S. H. Lee and S. H. Rhee:Efficient flooding for reliability in link-state routing protocols:2012 Intern ational Conference on ICT Convergence (ICTC). pp. 787-788(2012).

[17]T. Samant, P. Mukherjee, A. Mukherjee and A. Datta:TEEN - V: A solution for intra-cluster coope rative communication in wireless sensor network.2017 International Conference on I-SMAC (IoT in Soci al, Mobile, Analytics and Cloud) (I-SMAC). pp. 209-213(2017).

[18]D.E. Boubiche, S. Boubiche and A. Bilami:A Cross-Layer Watermarking-Based Mechanism for Dat a Aggregation Integrity in Heterogeneous WSNs.IEEE Communications Letters.pp. 823-826(2015).

[19]W. H. F. Aly:A novel dynamic secure combinatorial key approach for differentiated heterogeneous WSNs.2016 Wireless Days (WD). pp. 1-3(2016).

[20]S. Tanwar, S. Tyagi, N. Kumar and M. S. Obaidat:LA-MHR: Learning Automata Based Multilevel Heterogeneous Routing for Opportunistic Shared Spectrum Access to Enhance Lifetime of WSN. IEEE Systems Journal. pp. 313-323(2019) .

[21]P.L.R.Chze and K.S. Leong:A secure multi-hop routing for IoT communication.2014 IEEE World F orum on Internet of Things (WF-IoT). pp. 428-432(2014).

[22]Y. Chen, L. Chang and J. Ciou:A multi-hop distributed energy-efficient clustering architecture with sub-clustering in wireless sensor neworks.2016 International Conference on Machine Learning and Cybe rnetics (ICMLC). pp. 681-685(2016).

[23]P. Saini and A. K. Sharma:E-DEEC- Enhanced Distributed Energy Efficient Clustering scheme for h eterogeneous WSN.010 First International Conference On Parallel, Distributed and Grid Computing (PD GC 2010). pp. 205-210(2010).

[24]J.Wang, P. M. S. Carvalho and J. L. Kirtley:Improving Transformers' Utilization Under Single Conti ngency Policy and Customer Reliability Requirements.IEEE Transactions on Smart Grid. pp. 2384-2391 (2013) .

[25]Y. Zhao, L. T. Yang and R. Zhang:A Tensor-Based Multiple Clustering Approach With Its Applicati ons in Automation Systems.IEEE Transactions on Industrial Informatics.pp. 283-291(2018) .

[26]Y. Zhang, X. Zhang, S. Ning, J. Gao and Y. Liu:Energy-Efficient Multilevel Heterogeneous Routing Protocol for Wireless Sensor Networks.IEEE Access.pp. 55873-55884(2019).

[27]A. Verma, R. Mondal, P. Gupta and A. Kumar:Neural based Energy-Efficient Stable Clustering for Multilevel Heterogeneous WSNs.2018 First International Conference on Secure Cyber Computing and C ommunication (ICSCCC). pp. 208-212(2018).

[28]M. Bouraoui and A. Meddeb:Optimal number of cluster heads for random topology WSNs using the stable election protocol.2015 Global Summit on Computer \& Information Technology (GSCIT).pp. 1-5 (2015).

[29]A. Celik and A. E. Kamal:Multi-Objective Clustering Optimization for Multi-Channel Cooperative Spectrum Sensing in Heterogeneous Green CRNs.IEEE Transactions on Cognitive Communications and Networking.pp. 150-161( 2016).

[30]K. Li, G. Cheng, X. Sun and Z. Yang:A Nonlinear Flux Linkage Model for Bearingless Induction M otor Based on GWO-LSSVM.IEEE Access.pp. 36558-36567(2019).

[31]E. Emary, H. M. Zawbaa and C. Grosan:Experienced Gray Wolf Optimization Through Reinforcem ent Learning and Neural Networks.IEEE Transactions on Neural Networks and Learning Systems.pp. 68 1-694(2018).

[32]S. Arora, H. Singh, M. Sharma, S. Sharma and P. Anand:A New Hybrid Algorithm Based on Grey Wolf Optimization and Crow Search Algorithm for Unconstrained Function Optimization and Feature Se lection.IEEE Access pp. 26343-26361(2019). 
[33]Juang Chia-Feng,Lin Chan-Hung,Bui Trong Bac. Multiobjective Rule-Based Cooperative Continuou s Ant Colony Optimized Fuzzy Systems With a Robot Control Application.[J]. IEEE transactions on cyb ernetics,2020,50(2). 